\title{
Tibiofemoral Joint Forces during the Stance Phase of Gait after ACL Reconstruction
}

\author{
Brooke A. Sanford ${ }^{1 *}$, John L. Williams ${ }^{1}$, Audrey R. Zucker-Levin ${ }^{2}$, William M. Mihalko ${ }^{3}$ \\ ${ }^{1}$ Department of Biomedical Engineering, University of Memphis, Memphis, USA \\ ${ }^{2}$ Department of Physical Therapy, University of Tennessee Health Science Center, Memphis, USA \\ ${ }^{3}$ Campbell Clinic Department of Orthopaedics and Biomedical Engineering, \\ University of Tennessee Health Science Center, Memphis, USA \\ Email: *bwhitake@memphis.edu, john.williams@memphis.edu, azuckerl@uthsc.edu, wmihalko@campbellclinic.com
}

Received June 7, 2013; revised July 18, 2013; accepted August 20, 2013

Copyright (C 2013 Brooke A. Sanford et al. This is an open access article distributed under the Creative Commons Attribution License, which permits unrestricted use, distribution, and reproduction in any medium, provided the original work is properly cited.

\begin{abstract}
The main goals of anterior cruciate ligament reconstruction (ACLR) are to restore knee function and prevent development of osteoarthritis (OA). However, the incidence of early-onset OA remains higher in patients following ACLR. The purpose of this study was to compare the computed tibiofemoral joint (TFJ) forces and muscle forces of ACLR knees to those of BMI-matched control subjects during the stance phase of gait. We hypothesized that the use of principal component analysis would allow us to characterize alterations in three-dimensional TFJ loads and muscle forces after ACLR as compared to a healthy control population. Of the eight ACLR knees, four displayed an abnormal TFJ compressive force. In three of these four ACLR knees that displayed abnormal compressive forces, one of the major muscles/muscle groups crossing the knee also deviated from the control group. We believe that each subject has a unique response to their injury, reconstructive surgery, and rehabilitation.
\end{abstract}

Keywords: ACL Reconstruction; Tibiofemoral Joint Forces; Gait

\section{Introduction}

The main goals of anterior cruciate ligament reconstruction (ACLR) are to restore knee function and prevent development of osteoarthritis (OA). However, the incidence of early-onset OA remains higher in patients following ACLR [1]. It has been reported that abnormal motion and/or moments at the knee could lead to the development of early OA following ACLR [2,3]. To our knowledge, the tibiofemoral joint (TFJ) force during gait has not been studied as a possible contributory factor for OA development after ACLR.

Previous studies have reported TFJ loads for normal healthy individuals during gait [4,5]. Gardinier et al. [6] reported altered TFJ loading during gait in subjects who had sustained a complete ACL rupture within the past seven months. They found that patients walked with decreased force on their ACL deficient knee as compared to their contralateral knee. These results are contrary to a recent report that females who have undergone ACLR had increased tibiofemoral compressive forces during a single-leg drop-land task [7]. To our knowledge, there

*Corresponding author. are no reports describing TFJ loading during gait in subjects following ACLR.

We use principal component analysis (PCA) to explore differences between ACLR subjects' and control subjects' TFJ loads during the stance phase of gait. PCA is a dimension reduction method that transforms a set of observations of chosen variables that may be correlated with one another into a set of uncorrelated variables, called principal components [8,9]. PCA has previously been applied to gait waveform data to characterize differences among patients with a specific pathology and normal control subjects [10-13]. PCA allows consideration of the entire waveforms in the analysis as opposed to extracting arbitrary parameters.

The purpose of this study was to compare the TFJ forces and muscle forces of ACL reconstructed knees to those of BMI-matched control subjects during the stance phase of gait. We used the AnyBody Modeling System (Version 5.2, AnyBody Technology A/S, Aalborg, Denmark) to scale a musculoskeletal model to each individual and estimate tibiofemoral and muscle forces based on each individual's gait data. We hypothesized that the use of PCA would allow us to characterize alterations in 
three-dimensional TFJ loads and muscle forces after ACL reconstruction as compared to a healthy control population.

\section{Methods}

Sixteen subjects, eight (three male, five female) ACLR subjects (mean BMI 25.5, SD $4.1 \mathrm{~kg} / \mathrm{m}^{2}$ ) and eight BMImatched (five male, three female) control subjects with no history of lower extremity trauma were recruited (mean BMI 24.3, SD $4.3 \mathrm{~kg} / \mathrm{m}^{2}$ ) following institutional review board approval and informed consent. The ACLR subjects had sustained a unilateral ACL tear and seven were surgically reconstructed with patellar tendon grafts and one with a hamstring tendon graft more than seven months prior to testing (average 93 months). All subjects had a normal contralateral knee.

Prior to testing, retro-reflective markers were placed over bony landmarks including the sacrum, C7, and bilateral acromion, ASIS, PSIS, medial and lateral epicondyles, and medial and lateral malleoli, calcaneous, head of the fifth metatarsal, and dorsum of the foot. Arrays of markers were attached to the thighs and shanks using elastic wrap. A nine camera video-based opto-electronic system (Qualisys AB, Gothenburg, Sweden) was used for 3D motion capture as subjects walked barefoot at a selfselected speed on a 10 meter walkway instrumented with three force plates (AMTI, Watertown, MA, USA). Data were recorded for multiple trials to ensure that each foot made at least three clean footfalls on a force plate. Electromyogram (EMG) electrodes (Trigno, Delsys Inc., Boston, MA, USA) were placed bilaterally over the muscle belly of the rectus femoris, vastus medialis, biceps femoris, semitendinosus, and medial gastrocnemius. EMG data were collected at $2000 \mathrm{~Hz}$, rectified, low-pass filtered, and normalized by the maximum value during the stance phase.

Motion capture and force plate data were imported into the AnyBody Modeling System to estimate the TFJ forces for each subject [14] using the Twente Lower Extremity Model [15]. The model consists of 12 body segments: HAT (head, arms, and trunk), pelvis, and right and left femur, patella, tibia, talus, and foot. The model contains 11 joints: L5S1 and left and right hip, knee, patella/femur, talocrural and subtalar. The L5S1 and hip joints were modeled as a ball-and-socket, and the knee, talocrural, and subtalar joints were defined as a hinge. The patella could rotate with respect to the femur, but the orientation and position of the patella was dependent upon the knee flexion angle. The orientation and position of the pelvis with respect to the $3 \mathrm{D}$ global reference frame along with the joint rotations resulted in a model with 21 degrees of freedom. Each leg contained 56 muscles whose mechanical effect was modeled by 159 simple muscle slips, each consisting of a contractile element
[15].

The model was scaled in order to match each subject's anthropometry using a static, standing reference trial. The model was morphed using radial basis functions to match the assumed bony landmarks based on the marker positions [16]. An anthropometric data set [15] was used to model mass, inertia points, and muscle sites/geometry for all segments. The muscle attachment sites and geometries were scaled using a linear geometry scaling law. The muscle strength was scaled according to a length, mass, fat scaling which takes body mass index into account [17].

Inverse dynamics was performed and muscle forces were distributed by using a cubic polynomial optimization scheme that minimizes the sum of the cubes of muscle activations (force/maximum force) at each time step. The compression-distraction (CD), antero-posterior (AP), and medio-lateral (ML) TFJ force (in the tibial reference frame) during stance was averaged for each subject for three trials of gait for the ACLR knee. The same procedure was performed for the corresponding knees (right/ left) of BMI-matched control subjects. All gait waveforms were resampled to 101 values corresponding to $100 \%$ of the stance phase of gait (approximately 0\% $60 \%$ of the gait cycle). For each subject, force data were averaged from three trials for analysis.

We applied PCA to the averaged gait waveforms of control data to develop principal component (PC) models [10] for each measure, CD force, AP force, ML force, and gastrocnemius, vastii, rectus femoris, and hamstring muscle forces. Each PC model can be considered a projection of the data from the p-dimensional space defined by the original variables (101) to a k-dimensional hyperplane defined by the principal components, where $\mathrm{k}<\mathrm{p}$ $[9,10]$. The principal components are optimal in that they explain the maximal amount of variance in the original variables.

The principal component models were then used to reconstruct the original waveform of each control subject's knee, and the residual was calculated as the difference between the original and reconstructed waveforms. The sum of squares of the residuals, Q, was then calculated as a measure of the perpendicular distance of each knee from the hyper-plane defined by the PC model [10]. Similarly, the Mahalanobis distance, $\mathrm{T}^{2}$, is a measure of the distance of each knee from the center of the hyperplane. The Mahalanobis distance is a weighted sum of squares of the PC scores [10]. Upper limits for each of these measures, $\mathrm{T}^{2}$ and $\mathrm{Q}$, were derived from the normal subjects' data and used as a reference for comparing the ACLR data. Lower limits were not derived since both measures are squared quantities. Finally, the PC models developed from the control data were applied to the corresponding ACLR subject data, and values of $\mathrm{T}^{2}$ and $\mathrm{Q}$ 
were calculated for each ACLR knee for each gait measure. These values were compared to the control limits to determine deviation from normal.

\section{Results}

PC models were developed for the CD, AP, ML, gastrocnemius, vastii, rectus femoris, and hamstring forces (Table 1). The number of PCs used in each model (k) was chosen through an 85\% trace criterion [9]. Of the eight ACLR knees analyzed, five showed deviation in at least one of the TFJ forces (Table 2). In four of these five subjects, the abnormal TFJ force was accompanied by an alteration in one of the major muscles crossing the knee joint (hamstrings, quadriceps, and/or gastrocnemius).

The average tibiofemoral compressive force for both the ACLR and control groups was biphasic in shape, with the largest peak occurring during terminal stance and a lesser peak occurring at the end of the loading response (Figure 1(a)). Four ACLR subjects were identified as falling outside of the normal range for the $\mathrm{Q}$ value. The corresponding waveforms for these subjects show deviations from normal in magnitude (both increased and decreased), shape, and phase shift (Figure 1(b)).

The ML TFJ force was also biphasic in shape with peaks near the end of loading response and during terminal stance (Figure 2(a)). Again, four of the ACLR subjects had $\mathrm{Q}$ values which fell outside of the range defined by control subjects. As seen in Figure 2(b), the ACLR subjects deviated from normal in a variety of ways with no noticeable trend.

The mean AP TFJ force for both groups is shown in Figure 3. None of the ACL subjects had $\mathrm{T}^{2}$ or $\mathrm{Q}$ values above the normal range of control subjects.

Of the eight ACLR knees, four displayed an abnormal TFJ compressive force during stance. In three of these four ACLR knees that displayed abnormal compressive forces, one of the major muscles/muscle groups crossing the knee also deviated from the control group. Subject ACL4 displayed a TFJ compression force with a reduced magnitude from approximately $10 \%$ - $70 \%$ of the stance phase with a slightly delayed second peak (Figure 4(e)). When analyzing the corresponding muscle forces, the force in the vastii muscle group had a decreased magni-

Table 1. Principal component models.

\begin{tabular}{ccccccccc}
\hline Variation explained (\%) & \multicolumn{3}{c}{ Tibiofemoral joint force } & \multicolumn{3}{c}{ Muscle force } \\
\hline & CD & ML & AP & Gas & Vas & RF & Hams \\
PC1 & 53.7 & 57.9 & 69.6 & 67.9 & 82.5 & 65.4 & 58.1 \\
PC2 & 32.8 & 29.7 & 13.8 & 24.6 & 10.0 & 15.2 & 19.3 \\
PC3 & - & - & - & - & - & 8.9 & 13.4 \\
Total & 86.5 & 87.6 & 83.4 & 92.5 & 92.5 & 89.5 & 90.8 \\
\hline
\end{tabular}

The \% variation explained by each individual PC and total variation used in each model. Compression-distraction (CD), medio-lateral (ML), antero-posterior (AP), gastrocnemius (Gas), vastii (Vas), rectus femoris (RF), and hamstrings (Hams).

Table 2. ACLR subject assessment.

\begin{tabular}{|c|c|c|c|c|c|c|c|}
\hline \multirow[t]{2}{*}{ Subject (time since surgery, months) } & \multicolumn{3}{|c|}{ Tibiofemoral joint force } & \multicolumn{4}{|c|}{ Muscle force } \\
\hline & $\mathrm{CD}$ & ML & $\mathrm{AP}$ & Gas & Vast & $\mathrm{RF}$ & Hams \\
\hline ACL1 (7) & + & + & + & + & + & + & + \\
\hline ACL3 (64) & + & + & + & + & + & + & - \\
\hline ACL4 (15) & - & - & + & - & - & + & + \\
\hline ACL5 (87) & + & - & + & - & + & + & - \\
\hline ACL7 (232) & - & - & + & + & + & - & + \\
\hline ACL8 (143) & - & - & + & - & + & + & - \\
\hline ACL9 (157) & - & + & + & + & + & + & + \\
\hline $\operatorname{ACL}^{*} 0^{*}(42)$ & + & + & + & + & + & + & + \\
\hline Total significant & 4 & 4 & 0 & 3 & 1 & 1 & 3 \\
\hline
\end{tabular}

(-) indicates significantly different from normal either for $\mathrm{T}^{2}$ or $\mathrm{Q}$ at $95 \%$ confidence level. * ACL subject with hamstring graft. 


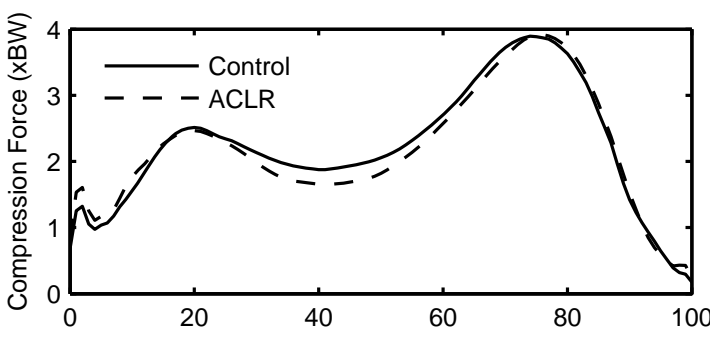

(a)

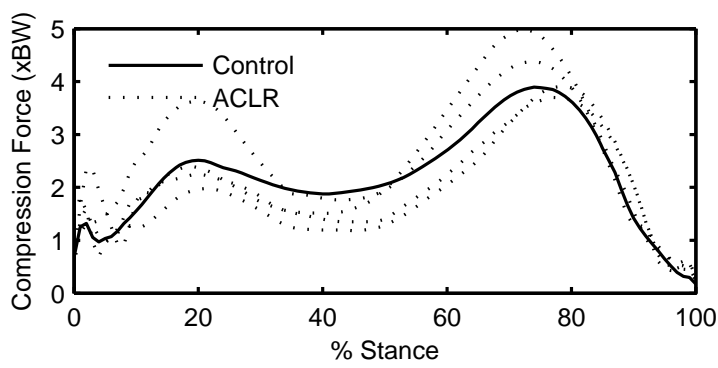

(b)

Figure 1. (a) Mean TFJ compression force for the control (solid) and ACLR (dashed) groups; (b) Four ACLR subjects with abnormal compression force (dashed) and mean for control group (solid).

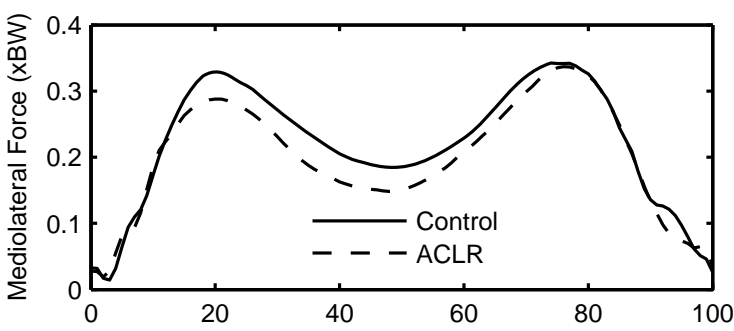

(a)

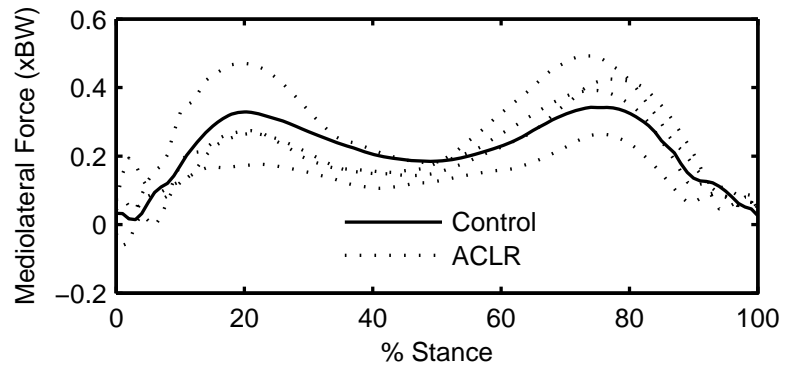

(b)

Figure 2. (a) Mean TFJ mediolateral force for the control (solid) and ACLR (dashed) groups; (b) Four ACLR subjects with abnormal mediolateral force (dashed) and mean for control group (solid).

tude from approximately $10 \%$ - $65 \%$ of the stance phase (Figure 4(b)) which was likely the cause of the reduced magnitude in the compression force. Also, the gastrocnemius force was delayed in time and slightly decreased in magnitude compared to controls (Figure 4(c)). The delay in gastrocnemius activation explains the delayed

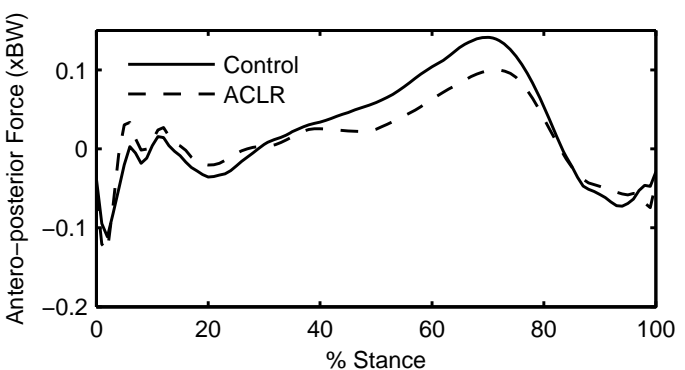

Figure 3. Mean TFJ antero-posterior force for the control (solid) and ACLR (dashed) groups.

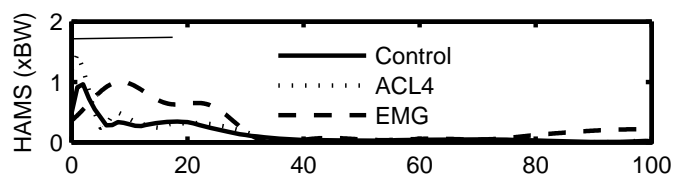

(a)

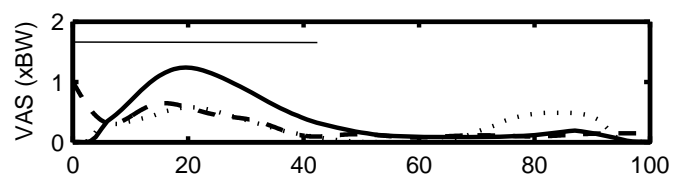

(b)

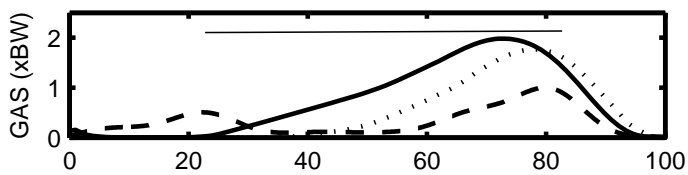

(c)

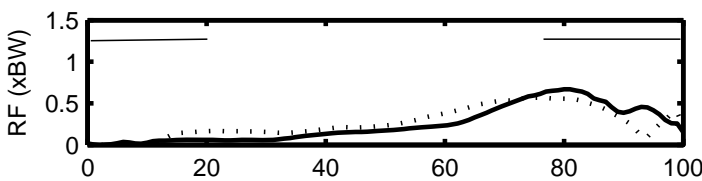

(d)

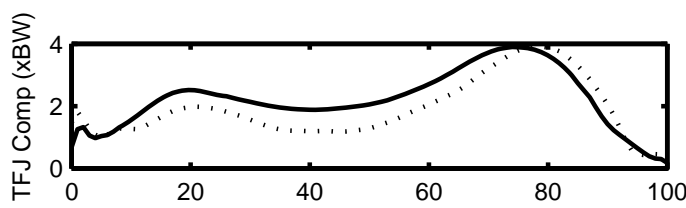

(e)

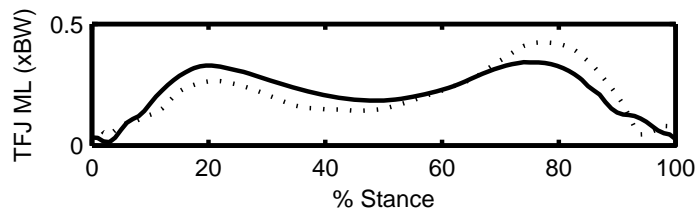

(f)

Figure 4. Predicted mean (a) hamstring force (b) vastii force (c) gastrocnemius force (d) rectus femoris force (e) TFJ compression force (f) TFJ medio-lateral force for the control group (solid) and subject ACL4 (dotted). EMG of the corresponding muscle (dashed) for subject ACL4 when available. Solid bars at top of plots represent normal EMG activity. 
second peak in the compression force.

Subject ACL7 displayed an abnormal TFJ compressive force which had increased first and second peaks during stance (Figure 5(e)). The rectus femoris muscle force was also found to lie outside the bounds of the normal control subjects and appears increased in magnitude from approximately $10 \%$ - 85\% of stance (Figure 5(d)).

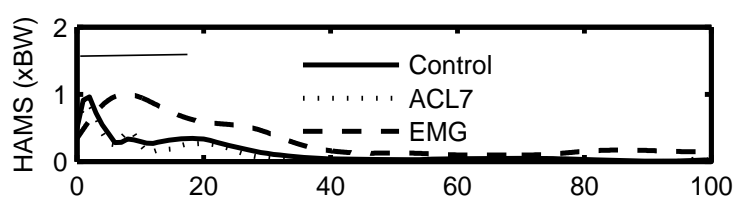

(a)

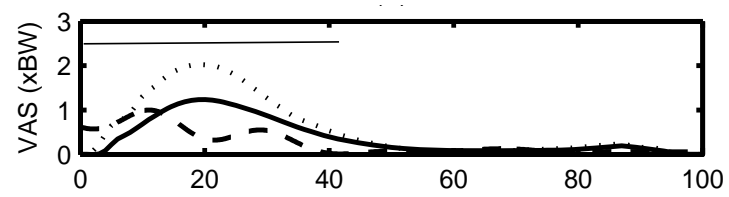

(b)

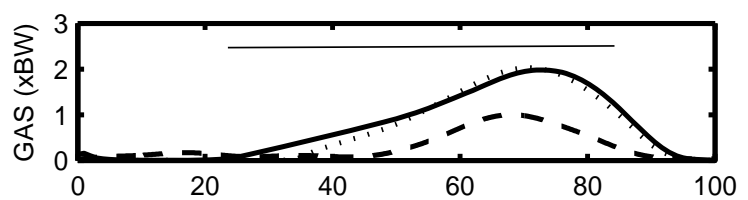

(c)

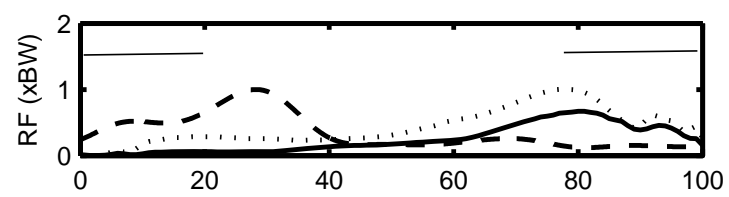

(d)

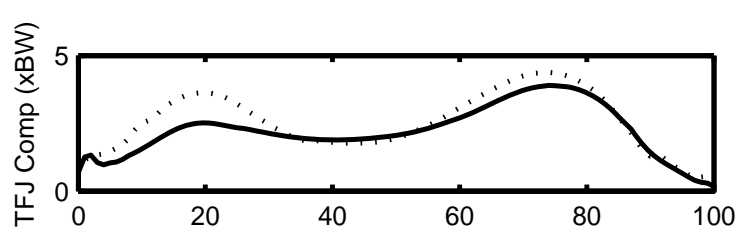

(e)

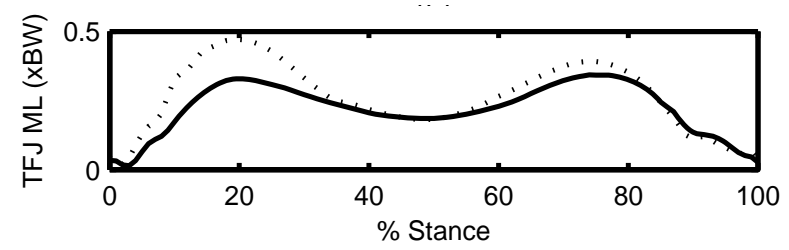

(f)

Figure 5. Predicted mean (a) hamstring force (b) vastii force (c) gastrocnemius force (d) rectus femoris force (e) TFJ compression force (f) TFJ medio-lateral force for the control group (solid) and subject ACL7 (dotted). EMG of the corresponding muscle (dashed) for subject ACL7 when available. Solid bars at top of plots represent normal EMG activity.
Subject ACL8 displayed a gastrocnemius force increased in magnitude at approximately $70 \%$ stance (Figure 6(c)) which is likely the cause for the increased TFJ compressive force which also occurs at $70 \%$ stance (Figure 6(e)). This subject's hamstring force was also considered abnormal when compared to control subjects (Figure 6(a)).

Finally, subject ACL9 had a compressive tibiofemoral force which deviated from that of the control group (Figure $7(\mathbf{e})$ ), but none of this subject's individual muscle

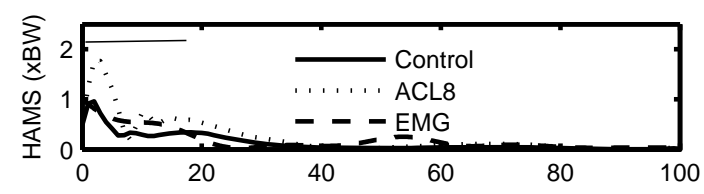

(a)

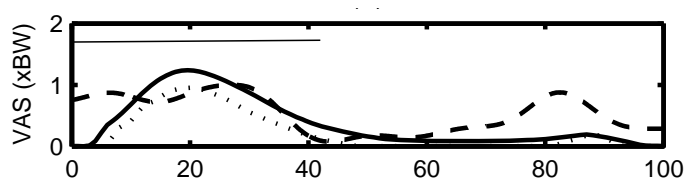

(b)

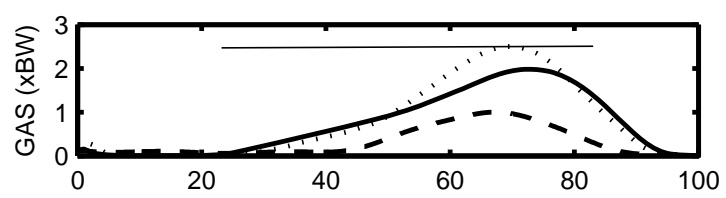

(c)

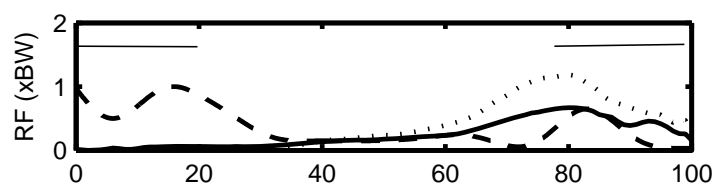

(d)

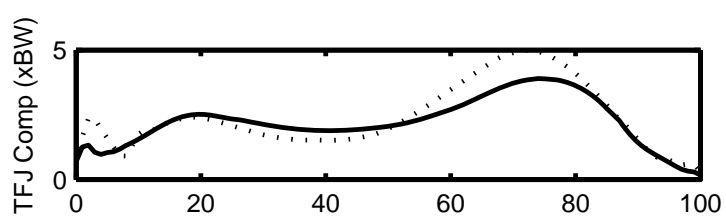

(e)

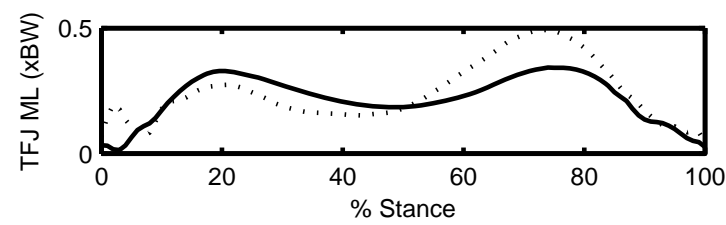

(f)

Figure 6. Predicted mean (a) hamstring force (b) vastii force (c) gastrocnemius force (d) rectus femoris force (e) TFJ compression force (f) TFJ medio-lateral force for the control group (solid) and subject ACL8 (dotted). EMG of the corresponding muscle (dashed) for subject ACL8 when available. Solid bars at top of plots represent normal EMG activity. 


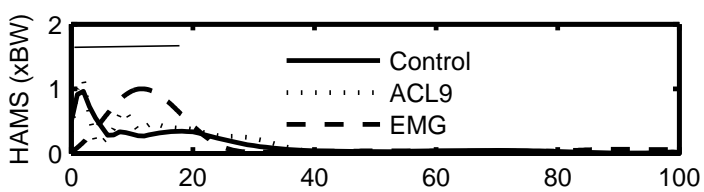

(a)

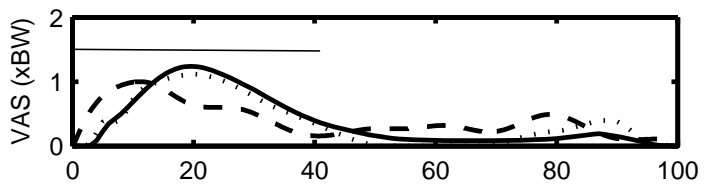

(b)

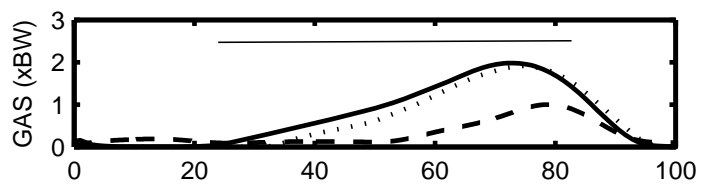

(c)

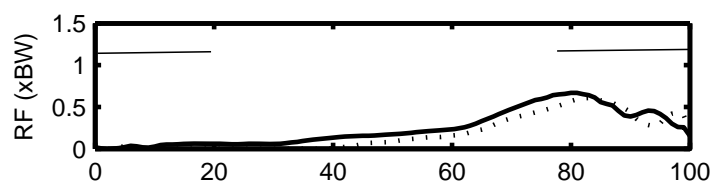

(d)

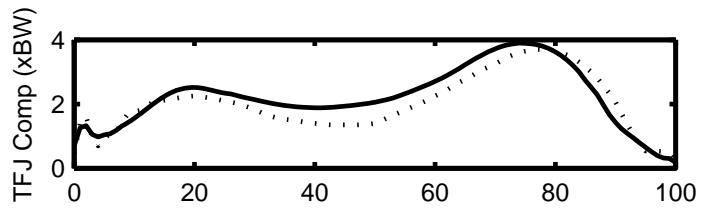

(e)

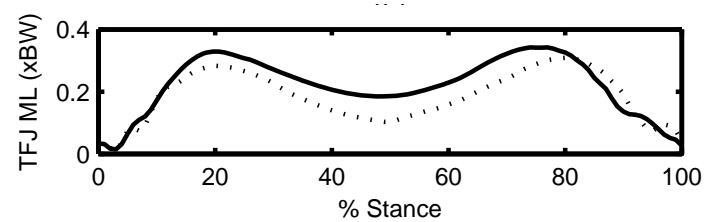

(f)

Figure 7. Predicted mean (a) hamstring force (b) vastii force (c) gastrocnemius force (d) rectus femoris force (e) TFJ compression force (f) TFJ medio-lateral force for the control group (solid) and subject ACL9 (dotted). EMG of the corresponding muscle (dashed) for subject ACL9 when available. Solid bars at top of plots represent normal EMG activity.

forces could explain the deviation in the compressive force (Figures 6(a)-(d)).

For the subjects who displayed abnormal TFJ forces, EMG data is plotted along with the muscle force to assess if the muscles in the model are active in the time that would be expected as determined from EMG. In cases where a muscle group force is shown, a representative muscle from that group was chosen from the available EMG data. EMG from the semitendinosus is plotted along with the hamstring muscle force, and the vastus- medialis EMG is plotted for the vastii muscle group. For the gastrocnemius force, EMG data was collected from the medial gastrocnemius. The EMG signal was not plotted if the signal to noise ratio was not at least four. Control subject EMG data for the same muscles is plotted below each graph to show the timing of normal muscle activity during the stance phase [18].

For the four ACL subjects shown, the predicted hamstrings muscle force activation coincides well with the timing of the semitendinosus EMG. The predicted vastii muscle force timing coincides well with EMG during the first half of stance phase, but some discrepancies were noted during the latter portion of stance in subjects ACL4 (Figure 4(b)) and ACL8 (Figure 6(b)). Subject ACL7 displayed rectus femoris EMG activity during the early part of stance, but the predicted muscle activity occurred predominantly later in stance (Figure 5(d)). Subject ACL8 displayed rectus femoris EMG activity both during early stance $(0 \%-35 \%)$ and later in stance $(70 \%$ $90 \%$ ), but the predicted muscle force only occurred during the latter portion of the stance phase (Figure 6(d)). Subject ACL4 showed a delay in both the predicted muscle activity and EMG of the gastrocnemius (Figure 4(c)). In the other three subjects, the timing of the predicted muscle force coincided with that of the control subjects but the gastrocnemius EMG was delayed slightly behind the predicted muscle force activity.

\section{Discussion}

We hypothesized that alterations in three-dimensional knee forces during the stance phase of gait could be detected as outliers following ACL reconstruction using PCA. The results show that abnormalities in gait waveforms can be detected in subjects who have undergone ACL reconstruction. Of the eight ACLR subjects analyzed, five were found to have an abnormality in either or both the CD and ML TFJ force. Four of these five subjects also had predicted muscle forces which deviated from those of the control population. The joint loading abnormalities appeared to be subject-specific, as there was variability across subject outcomes. This subjectspecific outcome is similar to what we found in our earlier work studying the knee joint moments during gait [13].

To our knowledge, this is the first report of knee joint forces and muscle forces during gait in subjects after ACL reconstruction. Tsai et al. [7] reported the peak TFJ compressive force in females who had undergone ACLR during a single-leg drop-land task. They found that the ACLR subjects had an increased peak compressive force when compared to a control group using an MRI-based EMG-driven model. We believe our results may differ due to the nature of the two different tasks, drop-land versus gait, and the modeling approach. Their model was 
EMG-driven, whereas ours was an inverse dynamics model. Also, their model only contained 10 muscles (hamstrings, quadriceps, and gastrocnemius). They did not incorporate the other muscles crossing the knee joint (popliteus, plantaris, gracilis, and sartorius) or other muscles which have been shown to affect tibiofemoral joint loading (i.e. soleus and gluteusmedius) [5]. Also, they do not show individual subject outcomes, so it is unclear how their subjects varied from normal on an individual basis.

It has been reported that acutely injured patients with ACL deficiency (ACLD) walk with decreased force on their injured knee compared to their uninjured knee [6]. This report was based on an EMG-driven model, and each subjects' ACLD knee was compared to the contralateral knee. Our study differs in that we compared the ACLR knees to knees of a healthy control population. Also, the subjects in the prior study [6] had all been injured within the past seven months before testing, whereas our subjects were an average of 93 months post-surgery. Whereas ACLD subjects in the prior study [6] walked with decreased force on the ACLD knee, we found that outcomes for our ACLR subjects were subject-specific in whether their TFJ forces were increased, decreased, or in the range of healthy control subjects. However, both studies report similar TFJ contact force patterns and magnitudes. The timing of muscle activation in our ACLR and control subjects were also similar to the ACL deficient subjects, although magnitudes could not be compared since they did not report the body mass for the representative set of muscle forces shown.

There are several limitations that need to be acknowledged. Firstly, the knee was modeled as a hinge joint which did not require muscles to balance the adductionabduction and internal-external rotation torques in the model. Secondly, the influence of ligaments, cartilage, fluid, and other soft tissues was not included in this model. However, the passive force contributions by the ligaments, etc. have been shown to be small [19] and would have likely had little influence on our results. Also, the muscle physiological cross-sectional areas and moment arms were scaled from cadaveric data based on each subject's mass and height since these values are difficult to measure in vivo. Predicted muscle forces have been shown to be sensitive to these parameters [20]. We also chose to distribute the muscle forces based on minimizing the sum of cubed muscle activations. Predictions of muscle force have also been shown to be sensitive to the optimality principle being applied [21]. The percentage of total muscle fibers activated (muscle activation) is related to muscle fatigue, and experimental evidence has shown this relationship to be approximately cubic [22]. Thus, while imperfect, we believe minimizing the sum of cubed muscle activation is a reasonable choice for the optimization criterion.

Finally, the sample sizes for both groups were relatively small. The results presented in this work were based on the assumption that the control dataset is truly representative of the normal population. Due to the small sample size we used to develop normal models and determine tolerance limits for comparison, appropriate caution must be exercised in interpreting the results.

\section{Conclusion}

Principal component analysis allowed us to identify TFJ loading waveforms and predicted muscle force waveforms which deviated from normal as determined using a control population. We found that of the eight ACLR knees, five knees displayed abnormal TFJ loading during the stance phase of gait. In four of these cases, the abnormal joint loading could be attributed to abnormal muscle forces of the major muscles crossing the knee joint. We believe that each subject has a unique response to their injury, reconstructive surgery, and rehabilitation. Further work is required to understand if this is due to surgical (e.g. graft orientation, placement, and tension) or patient variables (e.g. neuromuscular function, proprioception, and muscle strength) or both.

\section{Acknowledgements}

This work was funded in part by a grant from the Fed-Ex Institute of Technology to the University of Memphis.

\section{REFERENCES}

[1] L. S. Lohmander, P. M. Englund, L. L. Dahl and E. M. Roos, "The Long-Term Consequence of Anterior Cruciate Ligament and Meniscus Injuries," American Journal of Sports Medicine, Vol. 35, No. 10, 2007, pp. 17561769. http://dx.doi.org/10.1177/0363546507307396

[2] R. J. Butler, K. I. Minick, R. Ferber and F. Underwood, "Gait Mechanics after ACL Reconstruction: Implications for the Early Onset of Knee Osteoarthritis," British Journal of Sports Medicine, Vol. 43, No. 5, 2009, pp. 366-370. http://dx.doi.org/10.1136/bjsm.2008.052522

[3] M. Hall, C. A. Stevermer and J. C. Gillette, "Gait Analysis Post Anterior Cruciate Ligament Reconstruction: Knee Osteoarthritis Perspective," Gait Posture, Vol. 36, No. 1, 2012, pp. 56-60.

http://dx.doi.org/10.1016/j.gaitpost.2012.01.003

[4] P. Worsley, M. Stokes and M. Taylor, "Predicted Kinematics and Kinetics during Functional Activities Using Motion Capture and Musculoskeletal Modeling in Healthy Older People," Gait Posture, Vol. 33, No. 2, 2011, pp. 268-273. http://dx.doi.org/10.1016/j.gaitpost.2010.11.018

[5] P. Sritharan, Y. Lin and M. G. Pandy, "Muscles That Do Not Cross the Knee Contribute to the Knee Adduction Moment and Tibiofemoral Compartment Loading during Gait,” Journal of Orthoptera Research, Vol. 30, No. 10, 
2012, pp. 1586-1595. http://dx.doi.org/10.1002/jor.22082

[6] E. S. Gardinier, K. Manal, T. S. Buchanan and L. Snyder-Mackler, "Altered Loading in the Injured Knee after ACL Rupture,” Journal of Orthoptera Research, Vol. 31, No. 3, 2013, pp. 458-464. http://dx.doi.org/10.1002/jor.22249

[7] L. Tsai, S. McLean, P. M. Colletti and C. M. Powers, "Greater Muscle Con-Contraction Results in Increased Tibiofemoral Compressive Forces in Females Who Have Undergone Anterior Cruciate Ligament Reconstruction,” Journal of Orthoptera Research, Vol. 30, No. 12, 2012, pp. 2007-2014. http://dx.doi.org/10.1002/jor.22176

[8] J. E. Jackson, "Principal Components and Factor Analysis: Part I-Principal Components,” Journal of Quality Technology, Vol. 12, No. 4, 1980, pp. 201-213.

[9] J. E. Jackson, “A User’s Guide to Principal Components,” Wiley, Hoboken, 2003.

[10] K. J. Deluzio, U. P. Wyss, B. Zee, P. A. Costigan and C. Sorbie, "Principal Component Models of Knee Kinematics and Kinetics: Normal vs. Pathological Gait Patterns," Human Movement Science, Vol. 16, No. 2-3, 1997, pp. 201-217. http://dx.doi.org/10.1016/S0167-9457(96)00051-6

[11] K. J. Deluzio, U. P. Wyss, P. A. Costigan, C. Sorbie and B. Zee, "Gait Assessment in Unicompartmental Knee Arthroplasty Patients: Principal Component Modeling of Gait Waveforms and Clinical Status," Human Movement Science, Vol. 18, No. 5, 1999, pp. 701-711. http://dx.doi.org/10.1016/S0167-9457(99)00030-5

[12] K. J. Deluzio and J. L. Astephen, "Biomechanical Features of Gait Waveform Data Associated with Knee Osteoarthritis: An Application of Principal Component Analysis," Gait Posture, Vol. 25, 2007, pp. 86-93. http://dx.doi.org/10.1016/j.gaitpost.2006.01.007

[13] B. A. Sanford, A. R. Zucker-Levin, J. L. Williams, W. M. Mihalko and E. L. Jacobs, "Principal Component Analysis of Knee Kinematics and Kinetics after Anterior Cruciate Ligament Reconstruction," Gait Posture, Vol. 36, No. 3, 2012, pp. 609-613. http://dx.doi.org/10.1016/j.gaitpost.2012.06.003

[14] M. Damsgaard, J. Rasmussen, S. T. Christensen, E. Surma and M. de Zee, "Analysis of Musculoskeletal Systems in the AnyBodyModeling System," Simulation Modelling Practice and Theory, Vol. 14, No. 8, 2006, pp. 11001111. http://dx.doi.org/10.1016/j.simpat.2006.09.001

[15] M. D. Klein Horsman, H. F. J. M. Koopman, F. C. T. van der Helm, L. P. Prose and H. E. J. Veeger, "Morphological Muscle and Joint Parameters for Musculoskeletal Modeling of the Lower Extremity," Clinical Biomechanics, Vol. 22, No. 2, 2007, pp. 239-247. http://dx.doi.org/10.1016/j.clinbiomech.2006.10.003

[16] M. Lund, M. S. Anderson, M. de Zee and J. Rasmussen, "Functional Scaling of Musculoskeletal Models," Proceedings of the XXIII International Society of Biomechanics, Brussels, 2011.

[17] J. Rasmussen, M. de Zee, M. Damsgaard, S. T. Christensen, C. Marek and K. Siebertz, "A General Method for Scaling Musculo-Skeletal Models,” Proceedings of the International Symposium on Computer Simulation in Biomechanics, Cleveland, 2005.

[18] D. H. Sutherland, "Gait Disorders in Childhood and Adolescence,” Williams \& Wilkins, Baltimore, 1984.

[19] K. B. Shelburne, M. G. Pandy, F. C. Anderson and M. R. Torry, "Pattern of Anterior Cruciate Ligament Force in Normal Walking,” Journal of Biomechanics, Vol. 37, No. 6, 2004, pp. 797-805. http://dx.doi.org/10.1016/j.jbiomech.2003.10.010

[20] R. T. Raikova and B. I. Prilutsky, "Sensitivity of Predicted Muscle Forces to Parameters of the Optimization-Based Human Leg Model Revealed by Analytical and Numerical Analyses,” Journal of Biomechanics, Vol. 34, No. 10, 2001, pp. 1243-1255. http://dx.doi.org/10.1016/S0021-9290(01)00097-5

[21] M. Ackermann and A. van den Bogert, "Optimality Principles for Model-Based Prediction of Human Gait,” Journal of Biomechanics, Vol. 43, No. 6, 2010, pp. 10551060. http://dx.doi.org/10.1016/j.jbiomech.2009.12.012

[22] R. D. Crowninshield and R. A. Brand, “A Physiologically Based Criterion of Muscle Force Prediction in Locomotion,” Journal of Biomechanics, Vol. 14, No. 11, 1981, pp. 793-801.

http://dx.doi.org/10.1016/0021-9290(81)90035-X 